\title{
Elevated Neutrophil-to-Lymphocyte-ratio and Platelet-to-Lymphocyte Ratio in Myelofibrosis: Inflammatory Biomarkers or Representatives of Myeloproliferation Itself?
}

\author{
MARKO LUCIJANIC ${ }^{1}$, DAVID CICIC ${ }^{1}$, TAJANA STOOS-VEIC ${ }^{2,3}$, VLATKO PEJSA ${ }^{1,4}$, \\ JELENA LUCIJANIC ${ }^{5}$, AMINA FAZLIC DZANKIC ${ }^{1,6}$, JOSIPA VLASAC GLASNOVIC ${ }^{1}$, \\ ENA SORIC ${ }^{1}$, MARKO SKELIN ${ }^{7}$ and RAJKO KUSEC ${ }^{1,4,8}$ \\ ${ }^{1}$ Hematology Department, University Hospital Dubrava, Zagreb, Croatia; \\ ${ }^{2}$ Department of Clinical Cytology and Cytometry, University Hospital Dubrava, Zagreb, Croatia; \\ ${ }^{3}$ Faculty of Medicine, University of Osijek, Osijek, Croatia; \\ ${ }^{4}$ School of Medicine, University of Zagreb, Zagreb, Croatia; \\ ${ }^{5}$ Health Care Center Zagreb-West, Zagreb, Croatia; \\ ${ }^{6}$ Hematology Department, General Hospital Sisak, Sisak, Croatia; \\ ${ }^{7}$ Pharmacy Department, General Hospital Sibenik, Sibenik, Croatia; \\ ${ }^{8}$ Divison of Molecular Diagnosis and Genetics, Clinical Department of Laboratory Diagnostics, \\ University Hospital Dubrava, Zagreb, Croatia
}

\begin{abstract}
Background/Aim: We aimed to investigate clinical associations of inflammatory biomarkers neutrophil-tolymphocyte-ratio (NLR) and platelet-to-lymphocyte-ratio (PLR) in patients with myelofibrosis, myeloproliferative neoplasm with inflammatory background. Patients and Methods: We retrospectively analyzed a cohort of 102 myelofibrosis patients. $N L R$ and PLR were assessed in addition to other diseasespecific parameters. Results: NLR and PLR were significantly higher in myelofibrosis than in healthy controls. Higher NLR was significantly associated with Janus-kinase-2 (JAK2)mutation, wild-type-Calreticulin (CALR), older age and parameters reflecting increased proliferative potential of disease (higher leukocytes, higher hemoglobin, larger spleensize), whereas there was no significant association with $C$-reactive-protein (CRP). Higher PLR was significantly associated with absence of blast-phase-disease, absence of constitutional-symptoms, lower percentage-of-circulatoryblasts, smaller spleen-size and lower CRP. In the Coxregression-model, higher-NLR (HR=2.76; $p=0.004)$, lowerPLR (HR=1.99; $p=0.042)$ and Dynamic-International-
\end{abstract}

Correspondence to: Dr. Marko Lucijanic, Hematology Department, University hospital Dubrava, Av. Gojka Suska 6, 10000, Zagreb, Croatia. Tel: +38512902444. e-mail: markolucijanic@yahoo.com

Key Words: Philadelphia chromosome negative myeloproliferative neoplasm, primary myelofibrosis, secondary myelofibrosis, inflammatory biomarkers, survival.
Prognostic-System (DIPSS) $(H R=3.26 ; p<0.001)$ predicted inferior survival independently of each other. Conclusion: In the context of myelofibrosis, elevated NLR and PLR are more likely to represent myeloproliferation itself and not necessary the extent of inflammation.

Primary myelofibrosis (PMF) is a clonal disorder of hematopoietic stem cells (1) resulting in enhanced neoplastic myeloproliferation and increased inflammation. It is classified as a Philadelphia chromosome negative myeloproliferative neoplasm (Ph-MPN) together with polycythemia vera (PV) and essential thrombocytosis (ET) (2). Most of Ph- MPN patients bear somatic mutations in either Janus-kinase-2 $(J A K 2)$, calreticulin $(C A L R)$ or myeloproliferative-leukemiavirus-oncogene $(M P L)$ genes (3) that consequently activate JAK/signal-transducer-and-activator-of-transcription (STAT) signaling pathway, resulting in profound chronic inflammation $(4,5)$. PMF shows the most aggressive biological behavior among Ph- MPNs and bears the highest risk of disease progression and death (6). Clinical characteristics of PMF include proliferation or cytopenias of various myeloid lineages, elevated number of circulatory blasts, development of bone marrow fibrosis, extramedullary hematopoiesis in enlarged spleen and liver, and development of often debilitating constitutional symptoms. PV and ET patients can develop bone marrow fibrosis and clinical characteristics resembling PMF during the course of the disease when this condition is termed secondary myelofibrosis (SMF) (7). Risk of death in myelofibrosis 
patients is usually assessed using the International Prognostic Scoring System (IPSS) (8), the Dynamic IPSS (DIPSS) (9) and DIPSS-plus (10) that take age $>65$ years, hemoglobin $<100 \mathrm{~g} / 1$, white blood cells (WBC) $>25 \times 10^{9} / 1$, circulatory blasts $\geq 1 \%$ and presence of constitutional symptoms into account (IPSS at the time of diagnosis and DIPSS during course of the disease). DIPSS-plus additionally includes platelets $<100 \times 10^{9} / 1$, transfusion dependency and poor risk cytogenetics as adverse prognostic factors.

Neutrophil-to-lymphocyte-ratio (NLR) and platelet-tolymphocyte-ratio (PLR) are inflammatory biomarkers that have been consistently associated with poor clinical outcomes in a variety of malignant (11-15) and cardiovascular diseases (16-19) when elevated. Ph- MPNs are burdened with significant cardiovascular morbidity (20) and many predictors of decreased cardiovascular survival were shown to be prognostic in myelofibrosis as well (21-25). However, NLR and PLR have not been thoroughly studied in Ph- MPN patients so far. Therefore, we aimed to investigate clinical associations of NLR and PLR, and to assess their prognostic properties in the context of myelofibrosis.

\section{Patients and Methods}

A total of 102 patients with myelofibrosis who were evaluated in our institution in the period from 2006 to 2018 and were fulfilling 2016 WHO criteria (2) for PMF and IWG-MRT (7) criteria for SMF diagnosis were retrospectively analyzed. There were 80 (78.4\%) patients with PMF and $22(21.6 \%)$ patients with SMF. A total of $82(80.4 \%)$ patients were evaluated at the time of diagnosis and $20(19.6 \%)$ patients were evaluated at the time of referral. All procedures were in accordance with the ethical standards of the responsible committee on human experimentation (institutional or regional) and with the Helsinki Declaration of 1975, as revised in 1983. All patients provided written informed consent for molecular analyses. The study was approved by the Institutional Review Board.

Patients were staged according to the DIPSS prognostic scoring system (9). Spleen and liver size were assessed by palpation. Bone marrow fibrosis was graded according to the current European consensus (26). Hematological and disease specific clinical parameters were recorded (age, gender, WBC, differential blood count, circulatory blasts, hemoglobin level, mean corpuscular volume $(\mathrm{MCV})$, red cell distribution width (RDW), platelets, mean platelet volume (MPV), C reactive protein (CRP), lactate dehydrogenase $(\mathrm{LDH})$, serum albumin, serum iron, total iron binding capacity (TIBC), ferritin, transfusion dependency, presence of constitutional symptoms, blast phase disease, JAK2, CALR or MPL mutational status). NLR and PLR values of PMF and SMF patients were compared to 30 age and gender matched healthy controls.

Molecular analyses. For molecular analyses, DNA was isolated from blood by QIAamp DNA Blood Mini Kit (Qiagen, Hilden, Germany, ID 51104). JAK2 V617F was assessed by allele-specific PCR as described previously (27), CALRI and MPL exon 10 mutations were screened by high-resolution melting dye assays $(28,29)$ and any sample sequence that deviated from normal was Sanger sequenced.
Statistical methods. The normality of data distribution was tested using the Kolmogorov-Smirnov test. Numerical variables were presented as median and interquartile range (IQR), or as arithmetic mean \pm standard deviation depending on normality of data distribution. Categorical variables were presented as proportions. The Mann Whitney $U$-test, the Kruskal-Wallis test, the $\chi^{2}$ (Chi squared) test and the Spearman rank correlation were used where appropriate. Survival analyses (30) were performed using methods of Kaplan and Meier, the Cox-Mantel version of the log-rank test (31) and the Cox regression analysis. Receiver operating characteristic (ROC) curve analysis using survival status as a classification variable was performed for determining optimal cutoff values for survival analyses. $p$-Values $<0.05$ were considered statistically significant. Associations of different prognostic factors with survival were screened for using custom made MS Excel workbook (32). Analyses were performed using MedCalc Statistical Software version 18 (MedCalc Software BVBA, Ostend, Belgium).

\section{Results}

Patient characteristics. A total of 102 myelofibrosis patients were analyzed. Mean age was $65.7 \pm 10.8$ years, there were $61 / 102(59.8 \%)$ male patients. Patients' characteristics are shown in Table I.

Median follow-up of our cohort was 66 months. Median survival was 70 months, there was no difference in survival between PMF and SMF patients $(p=0.570)$.

Clinical associations of NLR. Median NLR in our myelofibrosis cohort was 4.8 IQR (2.7-8.2). NLR statistically significantly differed between diseased patients and controls $(p<0.001)$ : both PMF and SMF patients had similar NLR (median NLR $4.9 v s .4$ for PMF and SMF patients, respectively; $p=0.739$ ), and both PMF and SMF patients had higher NLR than healthy controls (median NLR 1.4 for controls, $p<0.05$ for both comparisons) as shown In Figure $1 \mathrm{~A}$.

Higher NLR values were statistically significantly associated with higher WBC count (Rho $0.54 ; p<0.001$ ), higher absolute neutrophil count (Rho $0.78 ; p<0.001$ ) and lower absolute lymphocyte count (Rho -0.25 ; $p=0.012$ ) as expected from NLR definition. In addition, higher NLR values were observed in $J A K 2$ mutated patients (median NLR $2.9 v s$. 6.4 for $J A K 2$ wild type and mutated patients, respectively; $p<0.001), C A L R$ wild type patients (median NLR 6.3 vs. 2.7 for $C A L R$ wild type and mutated patients, respectively; $p<0.001)$ and in patients without blast phase disease (median NLR 2.4 vs. 5 for patients with and without blast phase disease, respectively; $p=0.010)$. Higher NLR values were also statistically significantly associated with older age (Rho 0.27; $p=0.007$ ), higher hemoglobin (Rho $0.21 ; p=0.033)$, lower percentage of circulatory blasts (Rho $-0.22 ; p=0.026$ ), higher absolute monocyte count (Rho $0.47 ; p<0.001$ ), higher absolute basophil count (Rho 0.43; $p<0.001$ ), higher absolute eosinophil count (Rho 0.35; $p<0.001$ ), larger liver (Rho $0.21 ; p=0.039$ ), larger spleen 
Table I. Patient characteristics.

\begin{tabular}{|c|c|}
\hline Number of patients & 102 \\
\hline \multicolumn{2}{|l|}{ Diagnosis } \\
\hline PMF & $80 / 102(78.4 \%)$ \\
\hline SMF & $22 / 102(21.6 \%)$ \\
\hline Age (years) & $65.7 \pm 10.8$ \\
\hline \multicolumn{2}{|l|}{ Gender } \\
\hline Male & $61 / 102(59.8 \%)$ \\
\hline Female & $41 / 102(40.2 \%)$ \\
\hline \multicolumn{2}{|l|}{ Bone marrow fibrosis } \\
\hline Grade 0-I & $45 / 102(44.1 \%)$ \\
\hline Grade II-III & $57 / 102(55.9 \%)$ \\
\hline$J A K 2$ mutated & $60 / 99(60.6 \%)$ \\
\hline$C A L R$ mutated & $12 / 76(15.8 \%)$ \\
\hline$M P L$ mutated & $2 / 76(2.6 \%)$ \\
\hline Constitutional symptoms & $35 / 101(34.7 \%)$ \\
\hline Massive splenomegaly & $30 / 94(31.9 \%)$ \\
\hline Blast phase disease & $9 / 102(8.8 \%)$ \\
\hline WBC $\left(\times 10^{9} / 1\right)$ & 10.8 IQR (6.9-16.6) \\
\hline Absolute neutrophil count & 7.4 IQR (3.9-12.1) \\
\hline Absolute lymphocyte count & $1.5 \mathrm{IQR}(1-1.9)$ \\
\hline$\geq 1 \%$ circulatory blasts & $38 / 102(37.3 \%)$ \\
\hline Hemoglobin level (g/) & $114 \pm 25.2$ \\
\hline Platelets $\left(\times 10^{9} / 1\right)$ & 348.5 IQR (185.5-574) \\
\hline RDW (\%) & 19.5 IQR (17.6-21.2) \\
\hline LDH (U/1) & 538.5 IQR (336.8-758.5) \\
\hline $\mathrm{CRP}(\mathrm{mg} / \mathrm{l})$ & 4.7 IQR (2-14.1) \\
\hline NLR & $4.8 \mathrm{IQR}(2.7-8.2)$ \\
\hline PLR & 258 IQR (118-437.4) \\
\hline
\end{tabular}

PMF: Primary myelofibrosis; SMF: secondary myelofibrosis; JAK2: Janus kinase 2; CALR: calreticulin; MPL: myeloproliferative leukemia virus oncogene; WBC: white blood cells; IQR: interquartile range; RDW: red cell distribution width; LDH: lactate dehydrogenase; CRP: C reactive protein; NLR: neutrophil to lymphocyte ratio; PLR: platelet to lymphocyte ratio.

(Rho 0.21; $p=0.038$ ), lower serum iron (Rho -0.33; $p=0.002$ ), higher TIBC (Rho 0.22; $p=0.042$ ), lower transferrin saturation (Rho $-0.35 ; p=0.001$ ) and lower ferritin (Rho $-0.31 ; p=0.034)$. NLR showed no statistically significant association with degree of bone marrow fibrosis $(p=0.850)$, DIPSS risk category $(p=0.773), \mathrm{CRP}(p=0.448)$ or other tested parameters.

Clinical associations of PLR. Median PLR in our myelofibrosis cohort was 258 IQR (118-437.4). PLR statistically significantly differed between diseased patients and controls $(p<0.001)$ : both PMF and SMF patients had similar PLR (median PLR $258 \mathrm{vs.}$ 238.7 for PMF and SMF patients, respectively; $p=0.977$ ), and both PMF and SMF patients had higher PLR than healthy controls (median PLR 91.8 for controls, $p<0.05$ for both comparisons) as shown in Figure 1B.

Higher PLR values showed statistically significant association with higher platelets (Rho $0.78 ; p<0.001)$ and lower absolute lymphocyte count (Rho $-0.42 ; p<0.001$ ) as

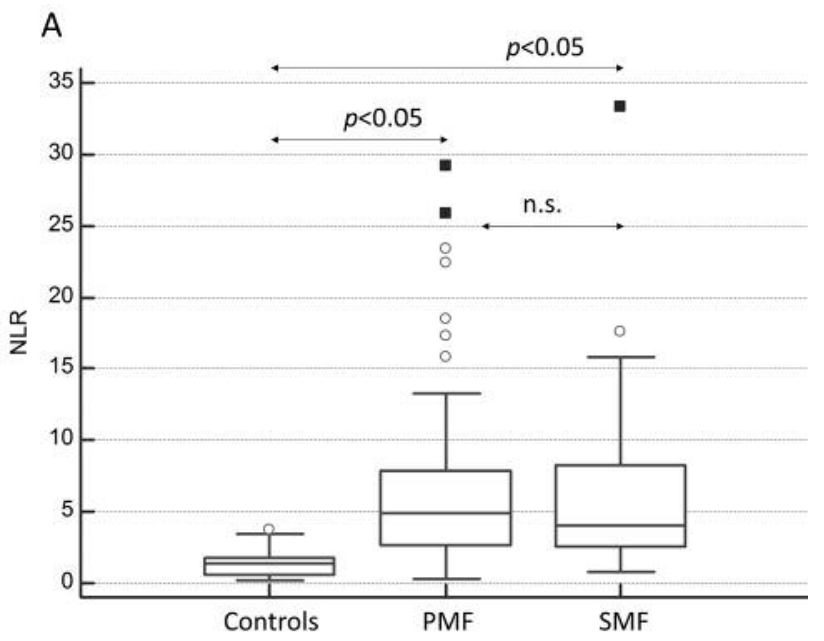

B

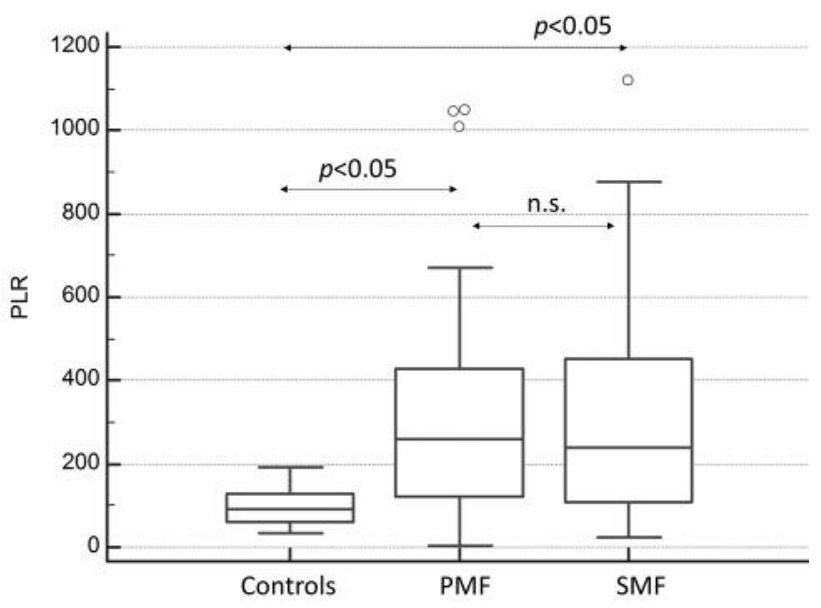

Figure 1. Both primary (PMF) and secondary myelofibrosis (SMF) patients presented with elevated A) neutrophil to lymphocyte ratio $(N L R)$ and $B)$ platelet to lymphocyte ratio $(P L R)$ in comparison to controls. n.s.: Non-significant.

expected from PLR definition. In addition, higher PLR values were observed in patients with an absence of blast phase disease (median PLR 59.4 vs. 269.4 for patients with and without blast phase disease, respectively; $p=0.010$ ) and patients with an absence of constitutional symptoms (median PLR $158.7 v s .277 .3$ for patients with and without constitutional symptoms, respectively; $p=0.015)$. Higher PLR values were also statistically significantly associated with lower percentage of circulatory blasts (Rho -0.32 ; $p=0.001$ ), lower spleen size (Rho $-0.24 ; p=0.021$ ), lower CRP (Rho $-0.33 ; p=0.002$ ), lower serum iron (Rho -0.29 ; $p=0.006$ ), higher TIBC (Rho 0.32; $p=0.003$ ), lower transferrin saturation (Rho $-0.34 ; p=0.001$ ), lower ferritin (Rho $-0.35 ; p=0.014$ ) and lower MPV (Rho $-0.3 ; p=0.002)$. PLR showed weak association with lower DIPSS risk 


\section{A) Overall survival by NLR}

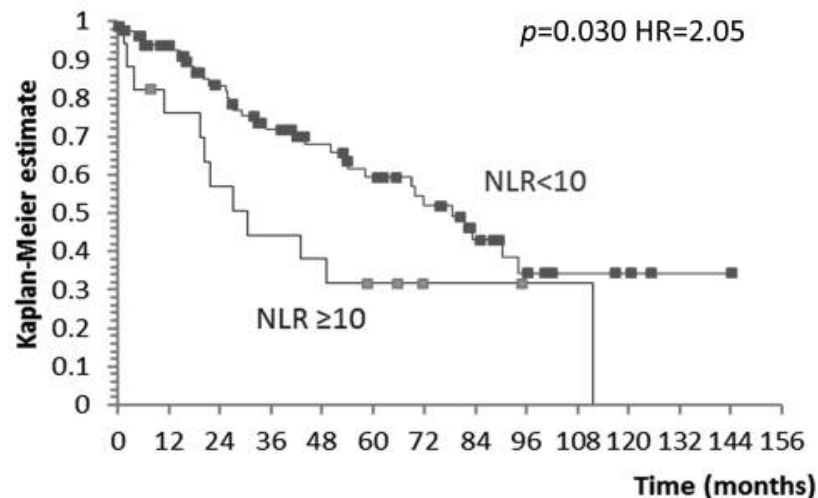

\section{B) Overall survival by PLR}

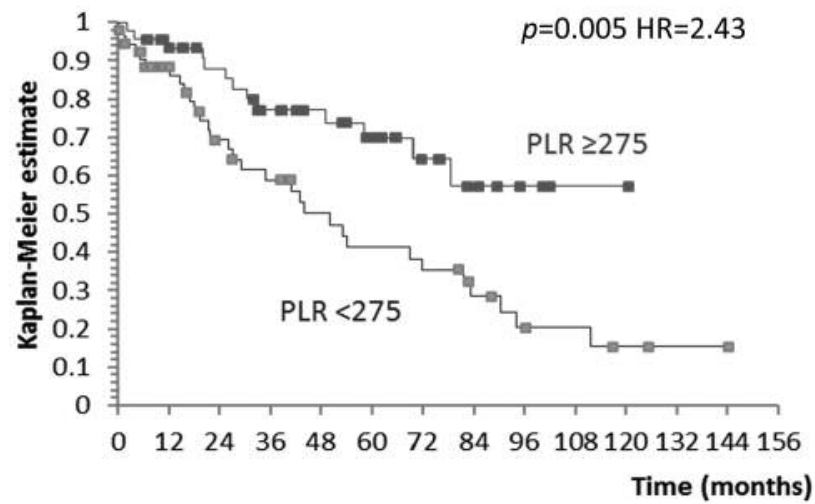

Figure 2. Overall survival of myelofibrosis patients stratified by A) neutrophil to lymphocyte ratio $(N L R)$ and $B)$ platelet to lymphocyte ratio (PLR). HR: Hazard ratio.

categories that was of borderline statistical significance (Rho $-0.2 ; p=0.052$ ). PLR showed no statistically significant association with any of the driver mutations $(p>0.05$ for associations with $J A K 2, C A L R$ and $M P L$ ), degree of bone marrow fibrosis $(p=0.240)$ or other tested parameters.

NLR and PLR were statistically significantly correlated (Rho $0.31 ; p=0.002$ ).

Negative prognostic implications of high NLR and low PLR. We determined optimal cut-off values for NLR and PLR for survival analyses using ROC-curve analysis and separated patients in groups with NLR $\geq 10$ (higher NLR) and NLR $<10$ (lower NLR) as well as PLR $\geq 275$ (higher PLR) and PLR $<275$ (lower PLR). In the univariate analyses, patients with myelofibrosis and higher NLR experienced shorter overall survival than patients with lower NLR (HR=2.05; $p=0.030$ ) as shown in Figure 2A. On the opposite, patients
Table II. Cox regression model comparing prognostic properties of neutrophil-to-lymphocyte ratio (NLR), platelet-to-lymphocyte ratio $(P L R)$, and the Dynamic International Prognostic Scoring System (DIPSS) contained variables.

\begin{tabular}{lll}
\hline & HR and 95\%CI & $p$-Value \\
\hline NLR $\geq 10$ & $3.57(1.48-8.58)$ & $0.005^{*}$ \\
PLR $<275$ & $2.34(1.18-4.63)$ & $0.014^{*}$ \\
Age $>65$ years & $1.22(0.61-2.43)$ & 0.577 \\
Hemoglobin $<100 \mathrm{~g} / 1$ & $3.56(1.66-7.62)$ & $0.001^{*}$ \\
WBC $>25 \times 10^{9} / 1$ & $2.53(1.14-5.65)$ & $0.023^{*}$ \\
$\geq 1 \%$ circulatory blasts & $2.46(1.29-4.72)$ & $0.007^{*}$ \\
Constitutional symptoms & $2.02(1.04-3.91)$ & $0.038^{*}$ \\
\hline
\end{tabular}

*Statistically significant at $p<0.05$. HR: Hazard ratio; 95\%CI: $95 \%$ confidence interval.

Table III. Cox regression model comparing prognostic properties of neutrophil-to-lymphocyte ratio $(N L R)$, platelet-to-lymphocyte ratio $(P L R)$, red cell distribution width $(R D W)$ and $C$ reactive protein $(C R P)$.

\begin{tabular}{llr}
\hline & \multicolumn{1}{c}{ HR and $95 \%$ CI } & $p$-Value \\
\hline NLR $\geq 10$ & $4.57(1.97-10.59)$ & $<0.001^{*}$ \\
PLR $<275$ & $3.55(1.64-7.72)$ & $0.001^{*}$ \\
RDW $>17.9$ & $2.81(1.06-7.49)$ & $0.039^{*}$ \\
CRP & $1.01(1-1.02)$ & $<0.001^{*}$ \\
\hline
\end{tabular}

* Statistically significant at $p<0.05$. HR: Hazard ratio; $95 \%$ CI: $95 \%$ confidence interval.

presenting with lower PLR experienced shorter overall survival in comparison to patients with higher PLR $(\mathrm{HR}=2.43 ; p=0.005)$ as shown in Figure 2B.

We performed a series of Cox regression models further investigating prognostic properties of NLR and PLR. We observed that both higher NLR (HR=2.43; $p=0.010)$ and lower PLR (HR=2.7; $p=0.003)$ remained statistically significant when analyzed together. These associations also remained statistically significant in the second Cox regression model additionally adjusted for DIPSS, where both higher NLR (HR=2.76; $p=0.004)$, lower PLR $(\mathrm{HR}=1.99 ; p=0.042)$ and DIPSS $(\mathrm{HR}=3.26 ; p<0.001)$ were able to predict poor survival independently of each other. We further explored how higher NLR and lower PLR perform when analyzed in the Cox regression model adjusted for particular DIPSS-contained components (Table II): all analyzed parameters except age remained statistically significant and could predict poor survival independently of each other suggesting that NLR and PLR provide additional prognostic information and have a potential for improvement in prognostication of myelofibrosis patients. 
In addition, we investigated prognostic properties of NLR and PLR in comparison to CRP and RDW that are inflammatory biomarkers with already recognized prognostic implications in PMF: all four parameters remained significantly associated with survival in the Cox regression model shown in Table III. Therefore, all four inflammatory biomarkers possess independent prognostic information and seem to represent different underlying pathophysiologic processes.

\section{Discussion}

To the best of our knowledge, our study is the first to investigate clinical associations and prognostic properties of NLR and PLR in patients with myelofibrosis, as well as to report unexpected findings of lower PLR as a negative prognostic parameter despite the high cardiovascular burden specific for the disease.

We could not identify prior studies investigating NLR or PLR in a population of myelofibrosis patients. However, two smaller studies assessed NLR/PLR in different Ph- MPN cohorts $(33,34)$. First study (33) suggested that NLR (among other inflammatory parameters) was elevated in a heterogenous cohort of Ph- MPN patients, which is in line with our findings of increased NLR in a population of myelofibrosis patients. NLR was not associated with history of thrombosis. Second study (34) investigated possible relationship between NLR and PLR and history of thrombosis in a cohort of PV and ET patients and found no significant association for both parameters as well. It is interesting to note that patients with a history of an unwanted event (prior thrombosis) had lower median PLR values (although difference did not reach statistical significance), a trend similar to our findings.

Increase in circulatory neutrophils and platelets due to neoplastic myeloproliferation, and concurrent decrease in circulatory lymphocytes $(35,36)$ due to unknown mechanisms, are typical findings in myelofibrosis. Since directions of changes in all three components favor rise in NLR and PLR, it is not surprising that these two parameters are elevated in comparison to healthy controls. However, in the context of myelofibrosis, higher WBC (8), but lower platelets (10) are recognized as negative prognostic parameters. This also seems to be reflected in prognostic associations of NLR and PLR since higher NLR, but lower PLR were predictive of poor survival.

Most of myelofibrosis patients die from complications of disease progression (bone marrow failure, leukemic transformation). These patients are also heavily burdened with cardiovascular comorbidities due to inflammation and accelerated atherosclerosis. Therefore, we initially expected opposite prognostic properties of PLR than observed. It should be noted that the lower PLR subgroup of myelofibrosis patients still had significantly higher PLR than healthy controls (data not shown). In addition, the lower PLR subgroup gathered patients with features of more advanced disease (leukemic transformation, larger spleen, higher percentage of circulatory blasts) and higher inflammatory status (higher CRP, more frequently present constitutional symptoms) which is reflected in higher mortality. It is evident however, that higher elevation in PLR does not represent stronger inflammation.

Higher NLR was associated with parameters reflecting higher proliferative potential of disease (JAK2 mutation, higher hemoglobin, higher WBC) and otherwise unfavorable features (older age, wild type CALR (37), higher monocyte and basophil counts (38)) which is reflected by higher mortality risk. However, NLR did not correlate with CRP or presence of constitutional symptoms. Interestingly, in the multivariate Cox regression model including higher NLR, lower PLR, CRP and RDW (all considered to represent inflammatory biomarkers), all four parameters remained statistically significant and predicted inferior survival independently of each other, further implying that they quantify different underlying pathophysiologic processes. In addition, both higher NLR and lower PLR predicted inferior survival independently of DIPSS and DIPSS containing components suggesting that NLR and PLR have good potential for improvement of prognostication in patients with myelofibrosis.

Limitations of our study include its retrospective design, single center experience, small number of patients and heterogenous study population. Nevertheless, our results provide interesting insight into the biology of myeloproliferative neoplasms and should be further explored in larger prospective cohorts of patients.

In conclusion, higher NLR, but lower PLR are associated with decreased survival and possess strong DIPSS independent prognostic properties. In the context of myelofibrosis, elevated NLR and PLR are more likely to represent myeloproliferation itself and not the extent of inflammation.

\section{Acknowledgements}

None.

\section{References}

1 Buschle M, Janssen JW, Drexler H, Lyons J, Anger B and Bartram CR: Evidence for pluripotent stem cell origin of idiopathic myelofibrosis: clonal analysis of a case characterized by a N-ras gene mutation. Leukemia 2: 658-660, 1988.

2 Arber DA, Orazi A, Hasserjian R, Thiele J, Borowitz MJ, Le Beau MM, Bloomfield CD, Cazzola M and Vardiman JW: The 2016 revision to the World Health Organization classification of myeloid neoplasms and acute leukemia. Blood 127: 2391-2405, 2016. 
3 Sliwa T, Beham-Schmid C, Burgstaller S, Buxhofer-Ausch V, Gastl G, Geissler K, Krauth M, Krippl P, Lang A, Petzer A, Wohrer S, Wolfler A and Gisslinger H: Austrian recommendations for the management of primary myelofibrosis, post-polycythemia vera myelofibrosis and post-essential thrombocythemia myelofibrosis: an expert statement. Wien Klin Wochenschr 129: 293-302, 2017.

4 Skoda RC, Duek A and Grisouard J: Pathogenesis of myeloproliferative neoplasms. Exp Hematol 43: 599-608, 2015.

5 Hasselbalch HC and Bjørn ME: MPNs as inflammatory diseases: The evidence, consequences, and perspectives. Mediators Inflamm 2015: 102476, 2015.

6 Hultcrantz M, Kristinsson SY, Andersson TM, Landgren O, Eloranta S, Derolf AR, Dickman PW and Bjorkholm M: Patterns of survival among patients with myeloproliferative neoplasms diagnosed in Sweden from 1973 to 2008: a population-based study. J Clin Oncol 30: 2995-3001, 2012.

7 Barosi G, Mesa RA, Thiele J, Cervantes F, Campbell PJ, Verstovsek S, Dupriez B, Levine RL, Passamonti F, Gotlib J, Reilly JT, Vannucchi AM, Hanson CA, Solberg LA, Orazi A, Tefferi A, International Working Group for Myelofibrosis Research and Treatment: Proposed criteria for the diagnosis of post-polycythemia vera and post-essential thrombocythemia myelofibrosis: a consensus statement from the International Working Group for Myelofibrosis Research and Treatment. Leukemia 22: 437-438, 2008.

8 Cervantes F, Dupriez B, Pereira A, Passamonti F, Reilly JT, Morra E, Vannucchi AM, Mesa RA, Demory JL, Barosi G, Rumi $\mathrm{E}$ and Tefferi A: New prognostic scoring system for primary myelofibrosis based on a study of the International Working Group for Myelofibrosis Research and Treatment. Blood 113: 2895-2901, 2009

9 Passamonti F, Cervantes F, Vannucchi AM, Morra E, Rumi E, Pereira A, Guglielmelli P, Pungolino E, Caramella M, Maffioli M, Pascutto C, Lazzarino M, Cazzola M and Tefferi A: A dynamic prognostic model to predict survival in primary myelofibrosis: a study by the IWG-MRT (International Working Group for Myeloproliferative Neoplasms Research and Treatment). Blood 115: 1703-1708, 2010.

10 Gangat N, Caramazza D, Vaidya R, George G, Begna K, Schwager S, Van Dyke D, Hanson C, Wu W, Pardanani A, Cervantes F, Passamonti F and Tefferi A: DIPSS plus: a refined Dynamic International Prognostic Scoring System for primary myelofibrosis that incorporates prognostic information from karyotype, platelet count, and transfusion status. J Clin Oncol 29: 392-397, 2011.

11 Periša V, Knezović A, Zibar L, Sinčić-Petričević J, Mjeda D, Periša I and Aurer I: Comparison of the prognostic impact of neutrophil/lymphocyte ratio, platelet/lymphocyte ratio, and Glasgow prognostic score in diffuse Large B-cell lymphoma. Shiraz E-Med J 17: e38209, 2016.

12 Szkandera J, Absenger G, Liegl-Atzwanger B, Pichler M, Stotz M, Samonigg H, Glehr M, Zacherl M, Stojakovic T, Gerger A and Leithner A: Elevated preoperative neutrophil/lymphocyte ratio is associated with poor prognosis in soft-tissue sarcoma patients. Br J Cancer 108: 1677-1683, 2013.

13 Wang DS, Ren C, Qiu MZ, Luo HY, Wang ZQ, Zhang DS, Wang FH, Li YH and Xu RH: Comparison of the prognostic value of various preoperative inflammation-based factors in patients with stage III gastric cancer. Tumour Biol 33: 749-756, 2012.
14 Wang DS, Luo HY, Qiu MZ, Wang ZQ, Zhang DS, Wang FH, $\mathrm{Li} \mathrm{YH}$ and $\mathrm{Xu} \mathrm{RH}$ : Comparison of the prognostic values of various inflammation based factors in patients with pancreatic cancer. Med Oncol 29: 3092-3100, 2012.

$15 \mathrm{He}$ CB and Lin XJ: Inflammation scores predict the survival of patients with hepatocellular carcinoma who were treated with transarterial chemoembolization and recombinant human type-5 adenovirus H101. PLoS One 12: e0174769, 2017.

16 Ming L, Jiang Z, Ma J, Wang Q, Wu F and Ping J: Platelet-tolymphocyte ratio, neutrophil-to-lymphocyte ratio, and platelet indices in patients with acute deep vein thrombosis. Vasa 47: 143-147, 2018

17 Wang Q, Ma J, Jiang Z and Ming L: Prognostic value of neutrophil-to-lymphocyte ratio and platelet-to-lymphocyte ratio in acute pulmonary embolism: a systematic review and metaanalysis. Int Angiol 37: 4-11, 2018.

18 Dong $\mathrm{CH}$, Wang ZM and Chen SY: Neutrophil to lymphocyte ratio predict mortality and major adverse cardiac events in acute coronary syndrome: A systematic review and meta-analysis. Clin Biochem 52: 131-136, 2018.

19 Durmus E, Kivrak T, Gerin F, Sunbul M, Sari I and Erdogan O: neutrophil-to-lymphocyte ratio and platelet-to-lymphocyte ratio are predictors of heart failure. Arq Bras Cardiol 105: 606-613, 2015.

20 Newberry KJ, Naqvi K, Nguyen KT, Cardenas-Turanzas M, Florencia Tanaka M, Pierce S and Verstovsek S: Comorbidities predict worse prognosis in patients with primary myelofibrosis. Cancer 120: 2996-3002, 2014.

21 Lucijanic M, Livun A, Tupek KM, Stoos-Veic T, Aralica G, Gecek I, Pejsa V and Kusec R: Heat shock protein 27 (HSP27/HSPB1) expression is increased in patients with primary and secondary myelofibrosis and may be affecting their survival. Leuk Lymphoma 58: 2497-2500, 2017.

22 Lucijanic M, Pejsa V, Jaksic O, Mitrovic Z, Tomasovic-Loncaric C, Stoos-Veic T, Prka Z, Pirsic M, Haris V, Vasilj T and Kusec $\mathrm{R}$ : The degree of anisocytosis predicts survival in patients with primary myelofibrosis. Acta Haematol 136: 98-100, 2016.

23 Barbui T, Carobbio A, Finazzi G, Guglielmelli P, Salmoiraghi S, Rosti V, Rambaldi A, Vannucchi AM and Barosi G: Elevated Creactive protein is associated with shortened leukemia-free survival in patients with myelofibrosis. Leukemia 27: 2084, 2013.

24 Lucijanic M, Mitrovic Z, Cicic D, Prka Z, Pejsa V, Livun A, Stoos-Veic T, Romic Z, Zivkovic M, Lucijanic I, Fabris Z and Kusec R: Increased mean platelet volume (MPV) is an independent predictor of inferior survival in patients with primary and secondary myelofibrosis. Int J Hematol 107: 166$172,2018$.

25 Lucijanic M, Prka Z, Pejsa V, Stoos-Veic T, Lucijanic J and Kusec R: Prognostic implications of low transferrin saturation in patients with primary myelofibrosis. Leuk Res 66: 89-95, 2018.

26 Thiele J, Kvasnicka HM, Facchetti F, Franco V, van der Walt J and Orazi A: European consensus on grading bone marrow fibrosis and assessment of cellularity. Haematologica 90: 11281132,2005

27 Baxter EJ, Scott LM, Campbell PJ, East C, Fourouclas N, Swanton S, Vassiliou GS, Bench AJ, Boyd EM, Curtin N, Scott MA, Erber WN, Green AR and Cancer Genome Project: Acquired mutation of the tyrosine kinase JAK2 in human myeloproliferative disorders. Lancet 365: 1054-1061, 2005. 
28 Bilbao-Sieyro C, Santana G, Moreno M, Torres L, SantanaLopez G, Rodriguez-Medina C, Perera M, Bellosillo B, de la Iglesia S, Molero T and Gomez-Casares MT: High resolution melting analysis: a rapid and accurate method to detect CALR mutations. PLoS One 9: e103511, 2014.

29 Pardanani A, Guglielmelli P, Lasho TL, Pancrazzi A, Finke CM, Vannucchi AM and Tefferi A: Primary myelofibrosis with or without mutant MPL: comparison of survival and clinical features involving 603 patients. Leukemia 25: 1834-1839, 2011.

30 Lucijanic $M$ and Petrovecki M: Analysis of censored data. Biochem Med 22: 151-155, 2012.

31 Lucijanic M, Skelin M and Lucijanic T: Survival analysis, more than meets the eye. Biochem Med 27: 14-18, 2017

32 Lucijanic M: Survival analysis in clinical practice: analyze your own data using an Excel workbook. Croat Med J 57: 77-79, 2016.

33 Hacibekiroglu T, Akinci S, Basturk A, Inal B, Guney T, Bakanay SM and Dilek I: Evaluation of inflammation parameters in Philadelphia negative chronic myeloproliferative neoplasia patients. Asian Pac J Cancer Prev 16: 5159-5162, 2015.

34 Kocak MZ, Dağlı M and Ünlü A: The ratio of platelet/ lymphocyte, the ratio of neutrophil/lymphocyte and some haemogram parameters related to thrombosis in essential thrombocytosis and polycythaemia vera. Biomed Res 28: 30363039, 2017.

35 Cervantes F, Hernandez-Boluda JC, Villamor N, Serra A and Montserrat E: Assessment of peripheral blood lymphocyte subsets in idiopathic myelofibrosis. Eur J Haematol 65: 104-108, 2000.
36 Lucijanic M, Veletic I, Rahelic D, Pejsa V, Cicic D, Skelin M, Livun A, Tupek KM, Stoos-Veic T, Lucijanic T, Maglicic A and Kusec R: Assessing serum albumin concentration, lymphocyte count and prognostic nutritional index might improve prognostication in patients with myelofibrosis. Wien Klin Wochenschr 130: 126-133, 2018.

37 Rumi E, Pietra D, Pascutto C, Guglielmelli P, Martinez-Trillos A, Casetti I, Colomer D, Pieri L, Pratcorona M, Rotunno G, Sant'Antonio E, Bellini M, Cavalloni C, Mannarelli C, Milanesi C, Boveri E, Ferretti V, Astori C, Rosti V, Cervantes F, Barosi G, Vannucchi AM, Cazzola M and Associazione Italiana per la Ricerca sul Cancro Gruppo Italiano Malattie Mieloproliferative Investigators: Clinical effect of driver mutations of JAK2, CALR, or MPL in primary myelofibrosis. Blood 124: 10621069,2014

38 Lucijanic M, Livun A, Stoos-Veic T, Pejsa V, Jaksic O, Cicic D, Lucijanic J, Romic Z, Orehovec B, Aralica G, Miletic M and Kusec R: High absolute basophil count is a powerful independent predictor of inferior overall survival in patients with primary myelofibrosis. Hematology, 2017. doi: 10.1080/ 10245332.2017.1376843. [Epub ahead of print]

Received February 28, 2018

Revised March 19, 2018

Accepted March 20, 2018 